\section{Affective neuroscience and psychiatry}

\author{
NEIL A. HARRISON and HUGO D. CRITCHLEY
}

\begin{abstract}
Summary Affective neuroscience addresses the brain mechanisms underlying emotional behaviour. In psychiatry, affective neuroscience finds application not only in understanding the neurobiology of mood disorders, but also by providing a framework for understanding the neural control of interpersonal and social behaviour and processes that underlie psychopathology. By providing a coherent conceptual framework, affective neuroscience is increasingly able to provide a mechanistic explanatory understanding of current therapies and is driving the development of novel therapeutic approaches.
\end{abstract}

\section{Declaration of interest None. Funding detailed in Acknowledgements.}

Over the past 25 years there has been a revolution within neuroscience, characterised by recognition of the importance of emotion to behaviour and subjective experience (Dalgleish, 2004). This revolution and the consequent snowballing of studies, now subsumed within affective neuroscience, was initiated and led by a key group of experimental, cognitive and social psychologists, anatomists, neuroscientists, clinical neurologists and psychiatrists. Affective neuroscience has challenged 'standard' cognitive models to account for empirical and clinical evidence of 'emotion' influencing, processes such as attention, perception, learning and memory at every level. Affective neuroscience focuses on brain function and how emotions are ultimately linked to genetic imperatives, primary motivations and reinforcement learning. More recently, affective neuroscience has extended its reach beyond the individual to address dynamic influences on social and economic behaviour.
Well-being and psychiatric dysfunction are necessarily measured against subjective emotional experience. Neurophysiological understanding of emotional disorders, including depression and bipolar disorder, provides a broad framework that may then usefully be applied to other psychiatric conditions, to address biological determinants of stress responses, disorders of personality and even prognosis across mental illness. This has contributed to a systematic integration of previously disparate genetic, neurochemical and psychodynamic models. This process continues to enrich the conceptual language empowering both clinician and patient and driving the development of novel diagnostic tools and therapeutic interventions. Neurobiological accounts of emotional behaviour, interpersonal and social interactions are increasingly plausible and no longer represent merely 'retreats into organicity' irreconcilable with psychosocial formulations. Affective neuroscience does not aim for a fully reductionist account of emotional social mechanisms, but rather to provide insights into control and influence of emotion without the constraints of established disciplinary boundaries. This may be illustrated in our increasing understanding of the neural processes mediating the effect of social and psychological stress on mood disturbance, memory impairments and enhanced risk of mortality. These cross-disciplinary approaches open exciting new treatment options.

\section{EMOTIONAL LEARNING}

To illustrate how advances within affective neuroscience have the potential to influence psychiatry, we have predictably chosen to focus our discussion around the 'most limbic of brain regions', the amygdala. The contribution of the amygdala to affective behaviour was recognised within MacLean's concepts of the visceral/limbic/mammalian brain (MacLean, 1990). Weiskrantz (1956), following observations by Klüver \& Bucy (1937) on behavioural effects of temporal lobectomy, showed that focal bilateral amygdala lesions had a detrimental impact on the social and emotional behaviour of monkeys. More recent studies of patients with bilateral amygdala lesions reveal impairments in processing of social and emotional cues, notably the recognition of facial or auditory expressions of fear (Adolphs et al, 1994). As a consequence, amygdala dysfunction is linked both theoretically and empirically to psychiatric disorders in which social behaviour is compromised. These include autism, schizophrenia and psychopathy. Practitioners can now conceptualise 'biological' impairments in emotional understanding/ behaviour as originating in focal dysfunction of regions such as the amygdala.

The amygdala supports the detection and learning of motivational significance. In animal experiments, this is typically illustrated in fear conditioning studies (i.e. the learning of threat). Fear conditioning represents a basic model for understanding the pathogenesis and maintenance of anxiety disorders, including post-traumatic stress disorder and panic. The role of amygdala in fear conditioning has been extensively described in rodents (LeDoux, 1996). In humans, the contribution of the amygdala to processing threat is evident in studies of patients with lesions and from neuroimaging, where amygdala activity is now almost treated as a biomarker of functional integrity within neural systems concerned with emotion. Findings such as these are now beginning to inform psychiatric treatment. Cognitive-behavioural therapy (CBT) remains a gold standard therapy for anxiety disorders by engendering 'unlearning' of fear responses through exposure and habituation. Animal studies indicate that both learning and unlearning (extinction) of threat are dependent on glutamine/ $N$-methyl-D-aspartic acid (NMDA) receptors within the amygdala dependent, a pharmacological mechanism that neuroscientists have stimulated to enhance this behavioural extinction process. Early trials suggest that boosting glutamine/ NMDA receptor function with cycloserine, a partial agonist at the NMDA receptor, enhances exposure-based CBT, with promising results in the treatment of acrophobia (fear of heights) and social anxiety (Hofmann et al, 2006). These findings result from a growing awareness of the important effects of emotional 
processes on basic cognitive functions and could not have resulted from models of memory based on standard cognitive neuroscience.

\section{SOCIAL BIOLOGY}

Recent widespread clinical recognition that pervasive neurodevelopmental disorders, including Asperger syndrome, place demands on clinical services for adults of working age have forced a reappraisal of conventional psychiatric practice. In parallel, neuroscience has explored the neural mechanisms through which we understand other people (i.e. the cognitive component of interpersonal interaction, which was previously a preserve of psychodynamic psychotherapy). Theoretically, we can understand the experience or intentions of other people by 'simulation'. Mirror neurons represent a convincing biological instantiation of simulation. Mirror neurons are located within premotor and motor cortex and in primates respond both when performing a specific action and when viewing another monkey performing that specific action (Rizzolatti et al, 1996). Many studies now show activity in the human brain, mimicking or mirroring the performance of perceived actions, which is consistent with a testable neural model for perspective taking, intentional stance and theory of mind. Emotional empathy has been related to co-activation of the same brain regions when experiencing actual physical pain and observing one's partner in pain (Singer et al, 2004). Similarly, brain responses to the distress of others engage unconscious autonomic bodily responses that mimic, in sympathy, the observed emotional cues (Harrison et al, 2006). Together these studies expand perception-action principles beyond classical 'mirror' regions and suggest that a correspondence between observed and experienced sensations, actions and feelings may be a more general feature of the human brain. Behavioural and neural evidence for robust simulatory systems actually predicts individual differences in emotional empathy. Conversely, individuals with autistic-spectrum disorders and developmental psychopathy show attenuated activation of brain 'mirror regions' when observing emotion in others. Such findings can have implications for the future diagnosis and monitoring of disorders of empathy.

\section{INTEGRATION}

The capacity for affective neuroscience to cross disciplines is illustrated within psychosomatic medicine, a field as relevant to primary care as liaison psychiatry. Broadly speaking, advances are being made in unpicking the mechanisms through which emotional trauma and stress impair cognitive, emotional and physical well-being. Adaptive physiological responses to acute physical and psychological trauma may have pathological effects on the brain and body if the challenges are extreme or prolonged. Stress hormones such as cortisol represent one mechanism. Lifelong stress (even within a general healthy population) is associated with reductions in hippocampal volume that reflect diminished cognitive (especially memory) and behavioural resources. Such findings offer a perspective on clinical psychiatry, as they address core processes underlying vulnerability to psychopathology. Psychosomatic medicine, within affective neuroscience, examines the health consequences of mind-body interactions. This has particular relevance to psychiatry where high rates of physical morbidity require appraisal in the context of potentially cardiotoxic and metabolic effects of medication.

\section{BROADENING THE NET}

Advances in methods for human brain imaging have assisted the affective neuroscience revolution. The capacity to observe the 'brain in action' at fairly high spatiotemporal resolution increasingly informs our understanding of physiological mechanisms underpinning human experience. In the clinical context, identification of functional and structural biomarkers contributes to the characterisation of psychiatric conditions and may enhance the monitoring of clinical course, and even targeting of treatments. Functional signatures in brain activity associated with depression and obsessive-compulsive disorder (OCD) were recognised relatively early. Subsequent studies of both healthy people and patients, using positron emission tomography and functional magnetic resonance imaging (Drevets et al, 1997), support the proposal that abnormalities in subgenual cingulate function in depression may relate to low mood and can predict treatment responsiveness. Animal studies had already linked this region closely with vegetative homoeostatic control. These findings, coupled with the observation of abnormalities of subgenual structure in individuals with unipolar depression, led to neurosurgical targeting of this region in treatment-resistant depression using deep brain stimulation resulting in a marked and sustained symptomatic improvement (Mayberg et al, 2005). Likewise, findings from neuroimaging studies are contributing to targeting of selective fronto-striato-thalamic circuits for the control of severe OCD and Tourette syndrome.

\section{PROSPECTS}

Biological psychiatry, particularly in relation to emotional disorder, is recovering from a position of low status within the hierarchy of scientific priorities. Affective neuroscience embodies a resurgence of interest in emotional behaviour within biological and social sciences. Recognition that emotion influences a broad spectrum of human functioning suggests that much of biological psychiatry could be rebranded clinical affective neuroscience. Integration of knowledge across disciplines represents an exciting future for psychiatry research and the examples emerging from affective neuroscience are promising. Molecular science already contributes to this integrative process (e.g. in studies of geneenvironment interactions which underpin emotional development, behaviour and psychiatric vulnerability). Consideration of biological, psychological and social aspects of psychiatric disorder is fundamental to clinical practice, and it is encouraging that these interactions are becoming central to both basic and clinical research.

Finally, affective neuroscience provides a robust investigative framework for exploring the fundamentals of adaptive emotional behavioural and psychiatric morbidity.

\section{ACKNOWLEDGEMENTS}

We thank Marcus Gray for helpful comments. N.A.H. and H.D.C. are funded by a Wellcome Trust programme grant to H.D.C.

\section{REFERENCES}

Adolphs, R., Tranel, D., Damasio, H., et al (1994) Impaired recognition of emotion in facial expressions following bilateral damage to the human amygdala. Nature, 372, 669-672 
Dalgleish, T. (2004) The emotional brain. Nature Reviews Neuroscience, 5, 582-589.

Drevets, W. C., Price, J. L., Simpson, J. R., et al (1997) Subgenual prefrontal cortex abnormalities in mood disorders. Nature, 386, 824-827.

Harrison, N. A., Singer, T., Rotshtein, P., et al (2006) Pupillary contagion: central mechanisms engaged in sadness processing. Social Cognitive and Affective Neuroscience, I, 5-17.

Hofmann, S. G., Meuret, A. E., Smits, J. A. J. et al (2006) Augmentation of exposure therapy with d-cycloserine for social anxiety disorders. Archives of General Psychiatry, 63, 298-304.

Kluver, H. \& Bucy, P. C. (1937) Psychic blindness and other symptoms following bilateral temporal lobectomy in Rhesus monkeys. American Journal of Physiology, II9, 352-353.

LeDoux, J. E. (1996) The Emotional Brain: The Mysterious Underpinnings of Emotional Life. Simon \& Schuster.

NEIL A. HARRISON, MRCP, MRCPsych, UCL Institute of Cognitive Neuroscience, London: HUGO D. CRITCHLEY, DPhil, MRCPsych, Brighton and Sussex Medical School, University of Sussex Campus, Brighton, UK

Correspondence: Professor Hugo Critchley, Department of Psychiatry, Brighton and Sussex Medical School, Univeristy of Sussex Campus, Falmer, Brighton BNI 3AR, UK. Email: h.critchley@bsms.ac.uk

(First received 16 February 2007, final revision 26 April 2007, accepted 3 May 2007)

MacLean, P. D. (1990) The Triune Brain in Evolution: Role in Paleocerebral Functions. Plenum.

Mayberg, H. S., Lozano, A. M., Voon, V., et al (2005)

Deep brain stimulation for treatment-resistant depression. Neuron, 45, 65I-660.

Rizzolatti, G., Fadiga, L., Gallese, V., et al (1996)

Premotor cortex and the recognition of motor actions. Brain Research Cognitive Brain Research, 3, |3|-|4|.
Singer, T., Seymour, B., O'Doherty, J., et al (2004)

Empathy for pain involves the affective but not sensory components of pain. Science, 303, II57-1162.

Weiskrantz, L. (1956) Behavioural changes associated with ablation of the amygdaloid complex in monkeys. Journal of Comparative Physiology and Psychology, 49, $381-391$. 